\title{
Zur Vorgeschichte der Königlichen Kabinets- ordre an Kant vom 1. Oktober 1794.
}

Neue Mitteilungen von Dr. Emil Fromm, Stadtbibliothekar in Aachen.

Kants Konflikt mit der preussischen Censurbehörde fällt in den Juni 1792. Seine "Religion innerhalb der Grenzen der blossen Vernunft", deren zweites Stück die Veranlassung zu dem Konflikt gegeben hatte, und in deren Vorrede er den für die Freiheit der Forschung bedeutangsvollen Streit mit der Censur fortsetzte, erschien dann zur Ostermesse 1793. Es wäre nicht verwunderlich gewesen, wenn man von Berlin aus sogleich mit irgend welchen Massnahmen gegen die Kühnheit des Philosophen vorgegangen wäre. Es geschah aber nichts, zunächst auch nicht als zu Ostern 1794 die zweite Auflage des gefährlichen Buches, wiederum bei Nicolovius in Königsberg, herauskam. Erst unterm 1. Oktober 1794 ist die von Woellner gezeichnete Königliche Kabinetsordre ergangen, welche Kant den Vorwurf machte, seine Philosophie „zu Ertstellung und Herabwürdigung mancher Haupt- und Grundlehren der heiligen Schrift und des Christentums“ namentlich in der "Religion innerhalb der Grenzen der blossen Vernunft", desgleichen in anderen kleineren Abhandlungen missbraucht za haben, und ihm zugleich befahl, sich künftighin nichts dergleichen zu Schulden kommen za lassen. Was, so müssen wir fragen, hat diesen „königlichen Spezialbefehl ${ }^{\star}$ unmittelbar herbeigeführt, wann ist der Beschluss zu einem Vorgehen gegen Kant gefasst worden und wer ist die treibende Kraft in diesem denkwürdigen Schanspiele gewesen?

In dem dritten seiner „Beiträge zu dem Material der Geschichte von Kants Leben und Schriftstellerthätigkeit in Bezug auf seine Religionslehre und seinen Konflikt mit der preussischen Regierung" hat Emil Arnoldt ${ }^{1}$ ) neuerdings versucht, diese Fragen zu beantworten, er hat aber dabei das Richtige meines Erachtens nicht getroffen. Denn nicht durch logische Kombinationen lassen sich die Fragen erledigen, sondern allein anf Grund des aktenmässigen Materiales. Zwar sind es nur wenige Notizen, welche über die Vorgeschichte der Kabinetsordre vom 1. Oktober sich in den Akten des Geheimen Staatsarchivs in Berlin vorfinden, sie sind aber wichtig genug, um ans Licht gezogen zu werden. ${ }^{2}$ )

1) Vgl. meine in dem Litteraturbericht dieses Heftes gegebene Besprechung der "Beiträge ${ }^{\mu}$.

2) Ich verdanke.den Nachweis der Notizen der Güte der Direktion des Kgl. Geheimen Staatsarchivs. 
Arnoldt legt Gewicht darauf, dass in dem Woellner'schen Reskripte neben der "Religion" auch kleinere Abhandlungen Kants als anstössig herangezogen sind, und er glanbt darnach suchen zu müssen, welche $\mathbf{A b}$ handlungen eigentlich gemeint seien und insbesondere welche der nach der ersten Auflage der "Religion" erschienenen Abhandlungen Woellner wohl zu dem entscheidenden Schritte gedrängt habe. Ich fasse seine Ausführungen zunächst kurz zusammen.

Der erste Aufsatz, welchen Kant unter Woellners Regiment in der Berlinischen Monatsschrift, im Septemberheft 1791, „tiber das Misslingen aller philosophischen Versuche in der Theodicee" erscheinen liess, war, wie Arnoldt im einzelnen ausführt, wohl geeignet, die Berliner Glaubensdespoten zu reizen. In der Schildernng der Freunde Hiobs, in der Schilderung ,ihrer Túcke ${ }^{\mu}$ durften Woellner und Hermes sich einen Spiegel vorgehalten und das Verdammungsurteil Gottes wider Hiobs Freunde auf sie selbst angewendet glauben; namentlich aber war die "Schlussanmerkung" des Aufsatzes unzweidentig auf den Gewissenszwang gemünzt, den Woellner and Hermes mit ihrem 1790 erlassenen "Schema examinis candidatorum" auszuäben versuchten. Auch die Abhandlung tber den Gemeinsprach: ${ }_{n}$ Das mag in der Theorie richtig sein, tangt aber nicht für die Praxis“, welche Kant zum Ersatz des von den Berliner Censoren zurückgewiesenen zweiten Stückes der philosophischen Religionslehre für die Berlinische Monatsschrift, im Septemberheft 1793, geliefert hatte, unterliess es nicht, dem Woellner'schen Regiment auf kirchlichem Gebiete nebenher zu opponieren, und trat hauptsächlich im zweiten and

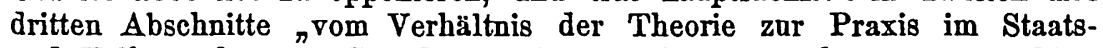
und Völkerrecht" mit Grundsätzen hervor, deren Anerkennung eine Verarteilung des bestehenden Regiernngssystems in Preussen wie in anderen Ländern Earopas nach sich ziehen musste. Auf diese Angriffe reagierte Woellner so wenig, wie auf das Erscheinen der "Religion innerhalb der Grenzen", obgleich der Druck gegen die Berliner Censurbehörde von Kant durchgesetzt worden war, auch die Publikation der zweiten Auflage der ${ }_{n}$ Religion" liess er ruhig geschehen; er schente sich vor dem Einschreiten wohl, wie Arnoldt meint, ${ }_{n}$ weil er die Folgen davon nicht absehen

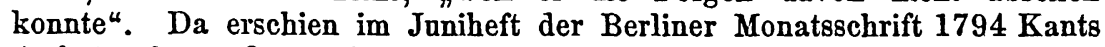
Aufsatz uber "das Ende aller Dinge!" Schon Kuno Fischer hat seine Darlegungen über diesen Aufsatz mit dem Hinweise abgeschlossen, dass man in der Schilderung der Urheber des widernatürlichen Weltendes die Züge der Woellner, Hillmer, Hermes, Woltersdorf n. a. erkennen und die Kantische Arbeit als ein auf das verkehrte Treiben des damaligen Zeitalters geworfenes grelles Schlaglicht ansehen könne. Dabei wird man Kant jedoch nicht die Absicht unterschieben müssen, dass er den Aufsatz lediglich zu dem Zwecke verfasst habe, gegen die thörichten Massregeln der Berliner Glaubenszuchtmeister von neuem Opposition zu machen. Auch der dritte Teil, welcher speziell als cin scharfer Angriff gegen Woellners Kirchenregiment angelegt und ansgestaltet ist, entbehrt nicht eines allgemeinen Charakters; aber die allgemeinen Gedanken, in denen sich die Auseinandersetzung fortbewegt, enthalten eben so viele bittere Anzüglichkeiten gegen jenes Regiment, und insgesamt stellen sie mit 
einer originellen Mischung von Treuherzigkeit und Ironie es als eine grosse Thorheit dar". Und nun, so argumentiert Arnoldt, war Kants Mass bei Woellner voll gemacht; der Aufsatz ther ${ }_{n}$ das Ende aller Dinge hat den Minister dahin gebracht, die Schale des Zornes, der sich bei ihm wider jenen angesammelt hatte, auszuschtitten. Also - erst im Juni 1794 wird ein Einschreiten gegen Kant beschlossen, und Woellner nebst seinen Genossen allein ist das bertichtigte Anklagerescript gegen den greisen Philosophen zazurechnon.

Die Dinge liegen in Wirklichkeit gänzlich anders!

Schon Martin Philippson hat in seiner „Geschichte des Prenssischen Staatswesens vom Tode Friedrich des Grossen bis zu den Freiheitskriegen", Bd. II, S. 81 darauf hingewiesen, dass in einer Sitzang der Geistlichen Immediat-Examinations-Kommission vom 2. April 1794 ausser Massnahmen gegen Niemeyer und Nösselt in Halle auch beschlossen worden sei, gegen den Frankfurter Professor Steinbart und gegen Hasse und Kant in Königsberg einzuschreiten. In dem Sitzungsberichte heisst es nun, was bei Philippson nicht steht, wörtlich: „Mit Steinbart, Hasse und Kant soll alles geschehen, was in der Zeitfolge die anzustellenden näheren Untersuchungen ergeben werden", und es unterliegt keinem Zweifel, dass dieser Beschluss der Immediat-Kommission direkt auf einen eigenhändigen Brief König Friedrich Wilhelms II. an Woellner d. d. Potsdam den 30. März 1794 zurückzufuhren ist. In diesem Briefe schreibt der König: „Za Frankfurt ist Steinbart, der anch da wird fortmüssen; zu Königsberg Hasse, der ein Haupt-Neologe ist; desgleichen mit Kantens schädlichen Schriften muss es auch nicht länger fortgehen." Das Schreiben schliesst dann mit den Worten: "Diesem Unwesen muss absolut gestenert werden, eher werden wir nicht wieder gate Freunde." 1) Gerade das Jahr 17.94 bildet, wie Paul Bailleu neuerdings ausgefuhrt hat, ${ }^{2}$ ) für die Stellung Woellners zum Könige einen Wendepunkt. „Durch die Teilnahme an dem Kriege gegen Frankreich, durch die Erwerbang Studpreassens war das Interesse des Königs an der Bekämpfung der Aufklarnng zeitweise abgelenkt, keineswegs erloschen. Als er im März 1794 von der Examinations-Kommission Berichte erhielt, welche die Erfolglosigkeit der bisherigen Massregeln einräumten, brauste sein Eigenwille hitzig auf." Er tadelte Woellner, der ein gelindes Verfahren za rechtfertigen suchte, in den schärfsten Ausdrücken, er nahm ihm das Ban-

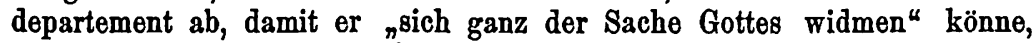
zugleich erliess er eine Reihe von Verfügungen, um ,in seinen Staaten ein rechtschaffenes thatiges Christentum als den Weg zar wahren Gottesfurcht aufrecht zn erhalten ${ }^{*}$ and verlangte von Woellner das energischste Vorgehen auf Grund der erlassenen Bestimmungen. Man sieht, so schliesst Bailleu seine Ausführungen, was als . Höhepunkt des Woellner'schen Regiments immer bezeichnet warde, ist thatsächlich ein ganz persönlicher Vorstoss des Königs in dem Kampfe gegen die Aufklärung, das letzte

1) Die Schlussworte mitgeteilt von P. Bailleu in dem Artikel ,Woellner" der Allgemeinen Deutschen Biographie, Bd. 44, Liefg. 1, 1898, S. 157.

2) Ebenda S. 156. 
Anfflackern seiner alten Kampfeslust, die mit der bald daranf eintretenden Abnahme seiner körperlichen nnd geistigen Kräfte gleichfalls allmăhlich verlöscht.

Gemäss den königlichen Weisungen wurde nun sogleich nach jener Sitzung der Immediat-Kommission vom 2. April mit den Massregeln gegen die Universităten begonnen. Unterm 15. April 1794 erging ein Reskript an Johann Gottfried Hasse als Verfasser einer im Jahre 1792 erschienenen Broschtre "tber jetzige und künftige Neologie" ; lass anch Kant an die Reihe kommen werde, muss bald daranf in weiteren Kreisen sich verbreitet haben. Kant selbst hielt seine Stellung bereits, als er die JuniAbhandlung an die Berlinische Monatsschrift übersandte, für bedroht; denn er schrieb am 18. Mai 1794 an Biester: ${ }^{1)}$ "Ich eile, hochgeschätzter Freund! Thnen die versprochene Abhandlung zu übersenden, ehe noch das Ende Threr und meiner Schriftstellerey eintritt .... Ich danke fur die mir ertheite Nachricht und uberzengt, jederzeit gewissenhatt and gesetzmässig gehandelt zu haben, sehe ich dem Ende dieser sonderbaren Veranstaltungen ruhig entgegen .... Das Leben ist kurz, vorneĥmlich das, was nach schon verlebten 70 Jahren übrig bleibt; um das sorgenfrey zu Ende za bringen, wird sich doch wohl ein Winkel der Erde ausfinden lassen." Im Mai oder Juni 1794 kam die Kunde, dass Kant seine Demission sogar schon erhalten habe, weil er einige Sätze in seinem Buche "Religion innerhalb der Grenzen der blossen Vernunft" nicht habe wider, rufen können and wollen, ${ }^{2}$ ) durch einen anonymen Brief nach Helmstedt an den Professor der Theologie, Abt H. Ph. K. Henke. Dieser besprach die Angelegenheit sogleich mit seinem Kollegen, dem Professor Heinrich Phil. Sextro, der den Plan, Kant nach Helmstedt za ziehen, schon früher mit dem die Universitätssachen in Braunschweig bearbeitenden Geheimen Justizrat Joh. Panl Mahner erwogen hatte. Sextro stellte in einem Schreiben vom 26. Juni 1794 Mahner jetzt anheim, den Herzog Karl Wilhelm Ferdinand von der Lage Kants und von der Möglichkeit, ihn für Helmstedt za gewinnen, za unterrichten. Zugleich trat auch der Vertreter der Philosophie in Helmstedt, Gottlob Ernst Schulze, der Verfasser des Aenesidemus, eifrigst für Kant ein; er richtete gleichfalls am 26. Juni an Mahner einen Brief, in welchem er ausfuhrte, dass es für die Universität ein sehr grosser Gewinn sein würde, wenn der bertihmte, und um die Philosophie wahrhaft nnsterblich verdiente Mann, unter die Lehrer auf

1) Mitgeteilt nach einem Excerpt in Reickes Kant-Briefsammlung von Arnoldt 8. 8. 0. S. 93.

2) Der Kommandant in Konigsberg, so lautete die Nachricht, hatte Kant zu sich kommen lassen und ihn betragt: ob er die in seinen Schriften vorgetragenen Meinungen widerrufen wolle oder nicht? ${ }_{n}$ Er møgte darauf nur sogleich mit ja oder nein antworten. Als nun Kant erklärte, er würde nichts widerrufen, so hat ihm der Kommandant gesagt, er erhalte hiermit die Entlassung von seinem Amte als Professor der Philosophie in Königsberg" (vgl. Pa al Zimmermann im Braunschweigischen Magazin, Bd. 2, Braunschweig 1896, S. 172t., wo die geplante Berufung Kants nach Helmstedt zum ersten Male eingehend behandelt ist; die interessanten Mitteilungen Zimmermanns sind Arnoldt entgangen, sie sind aber auch sonst in der Kant-Litteratur bisher unbeachtet geblieben). 
derselben gehörte, und dass er trotz seiner. bekannten Stellung zur Kantischen Philosophie gern dazn beitragen möchte, „dass der grosse Mann unserer Akademie za Teil würde und sein Leben unter dem Schatze eines Fursten beschlösse, in dessen Staate die verfolgte Philosophie, wie einst in dem Staate Friedrich des Grossen, Sicherheit gegen den Aberglauben und die Unwissenheit findet." Mahner hat die Angelegenheit sogleich bei dem Herzoge zur Sprache gebracht, jedoch nicht mit Erfolg. In einem eigenhändigen Schreiben vom 28. Juni führte der Herzog ablehnend aus, dass die Berufung nichts gegen sich haben wirde, $n$ wurden nar nicht die Meinungen uber theologische und philosophische Gegenstände in jetzigen Zeiten, wo ganz Europa gespannet ist, und wo selbst die mächtigsten Regierungen in Besorgnis schweben, als die Quellen der unabsehnlichen Unruhen betrachtet" ; aus politischen Rücksichten, namentlich um sich nicht in einen offenbaren Gegensatz za Preussen za stellen, glanbe er von dem Antrag absehen za müssen. Am 1. Juli antwortete daher Mahner den Helmstedter Professoren, „dass Serenissimus aus politischen Rücksichten bedenklich fänden, auf eine Vocation Kants einzugehen."

Inzwischen hatte Hasse ein würdeloses Rechtfertigungsschreiben bei Woellner eingereicht, worauf unterm 19. August 1794 von Berlin aus verfügt wurde, dass er sich in seinem mündlichen Untericht and in seinen Schriften genau nach dem Religionsedikt zu richten habe, widrigenfalls unfehlbar strengere Verfügungen gegen ihn ergehen würden. Gleichzeitig war eine Untersuchung über Ausschreitungen geführt worden, welche Studenten angeblich in einer der Königsberger Kirchen verübt hatten. Die dem Gebiete der Komik angehörige Angelegenheit 1) fand ihren Abschluss durch ein Reskript Woellners vom 30. September 1794; das Datnm des folgenden Tages trägt die Kabinetsordre an Kant. Der Unterschrift Woellners waren die Worte vorangestellt: „Auf Seiner Königl. Majestät allergnädigsten Specialbefehl" ; mochten solche Worte auch sonst nur einen formellen Wert haben, hier bezeichneten sie genau den Thatsachen entsprechend die Entstehung des Reskriptes.

Ich fasse das Gesagte zusammen: Die Zurückweisung des 2. Stückes der Religionsschrift durch die Berliner Censur und die von Kant gegen die Berliner Censur durchgesetzte Veröffentlichung des vollständigen Buches, das sind die Thatsachen, welche die Schriftstellerei des Philosophen dem König Friedrich Wilhelm II. als gefährlich erscheinen liessen. Dieser Schriftstellerei sollte ein Ziel gesetzt werden, und man braucht daher, wenn Friedrich Wilhelm von "Kantens schädlichen Schriften" spricht, nicht etwa danach zu suchen, welche kleinere Abhandlungen er ausser der "Religion" noch im Auge gehabt haben könne. Dass nicht sogleich nach dem Erscheinen der ersten Auflage der "Religion" eingeschritten worden ist, war durch äussere Umstände bedingt. Der König selbst ist die treibende Kraft in der energischen Bekämpfung der "Anfklärung", und sobald die politischen Ereignisse ihm wieder Zeit und

1) Näheres über diese Dinge findet sich in meiner Schrift „Immanuel Kant und die preussische Censur" (Hamburg u. Leipzig 1894) S. 45 ff. 
Musse für diese Dinge liessen, hat er durch persönliches Eingreifen auch die Massnahmen gegen Kant herrorgerufen.1) Sie sind am 2. April 1794, wo in der Immediat-Kommission das eigenhändige Schreiben des Königs zur Beratung gestanden hatte, eine beschlossene Sache. Dass Woellner dann bei der Abfassung des Reskriptes vom 1. Oktober 1794 sich auch über die in den letzten Jahren erschienenen kleineren Abhandlungen Kants unterrichtet haben wird, ist allerdings begreiflich und wabrscheinlich und ebenso, dass unter diesen Abhandlungen der Juni-Aufsatz 1794 seinen besonderen Unwillen erregt haben mag. Um seinetwillen hat er vielleicht die kleineren Arbeiten Kants in seiner Verfügung noch besonders herangezogen, darin mag Arnoldt Recht haben. Hinfällig hingegen sind Arnoldts Ausführungen, ${ }^{2}$ ) soweit sie Woellner allein die Schuld an dem Vorgehen gegen Kant beimessen und die Massregelung erst durch den Juni-Aufsatz über ${ }_{n}$ das Ende aller Dinge" direkt veranlasst sehen.

1) Ich weise gleich hier eine Unterstellung zurïck, welche Arnoldt in dem Vorwort seiner "Beiträges (vgl. unten) S. IV Anmerk. gegen mich gemacht hat. Er spricht da mit offenbarer Beziehung auf meine eben zitierte Schrift von "Kants auch schönfïrbender Charakteristik" Friedrich Wilhelms II., die aber doch eine leise - freilich viel zu leise - Anspielung auf das lästerliche Privatleben des Königs enthalte. Ich habe in meiner Schrift S. 18, Anm. 2 das Buch von Paulus Cassel eine ungeschickte und konfuse Verteidigungsschrift des Königg genannt und damit meine Auffassung über Friedrich Wilhelm genligend gekennzeichnet. Gefärbt zu Gunsten des Königs habe ich dort so wenig irgend etwas, wie ich es hier thue; ich habe nur verschmäht, mich mit der liederlichen Gesellschaft, die damals in den Königlichen Schlössern dominierte, behaglich zu befassen, weil das nicht zur Sache gehörte. Gerade das scheint Arnoldt aber besonders übel vermerkt zu haben.

2) Auch Paulsen in seinem neuen Kantbuch S. 366 schliesst sich denselben noch an. 